\title{
Promoción de la investigación de alta calidad en temas prioritarios para la salud en América Latina y el Caribe*
}

\author{
Fabio Zicker ${ }^{1}$, Luis Gabriel Cuervo ${ }^{2}$ y Luis Alejandro Salicrup ${ }^{3}$
}

\author{
La región aún tiene que fortalecer su capacidad de investigación con el fin de fundamentar \\ políticas y prácticas eficaces de salud pública, sostienen Fabio Zicker y cols.
}

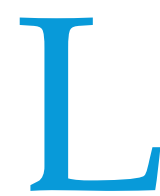

a evidencia científica procedente de estudios de investigación de alta calidad es importante para mejorar la salud mundial y la equidad en la salud. ${ }^{1}$ En los entornos con recursos limitados, son esenciales los conocimientos y las herramientas que sean pertinentes a escala regional a fin de reforzar la efectividad de los servicios de salud y reducir la carga de enfermedad. ${ }^{2}$

La creación de sistemas nacionales de investigación en salud que se centren en las prioridades locales en América Latina y el Caribe puede facilitar la investigación de alta calidad y fortalecer la capacidad de realizarla. ${ }^{3}$ Durante los últimos 15 años, el gasto general en investigación y desarrollo en la región ha aumentado de $0,57 \%$ a $0,8 \%$ del producto interno bruto (PIB). Sin embargo, esta cifra es todavía muy inferior al nivel de la inversión en los países de la Organización de Cooperación y Desarrollo Económicos $(2,5 \%$ a 2,8\% del PIB). ${ }^{4}$

Aunque el número de publicaciones en ciencias de la salud provenientes de América Latina y el Caribe que pueden citarse ha triplicado, ${ }^{5}$ aún es necesario mejorar

\footnotetext{
* Traducción oficial al español efectuada por la Organización Panamericana de la Salud. En caso de discrepancia, prevalecerá la versión original en inglés.

1 Especialista en Ciencia, Tecnología e Innovación de la Salud Mundial. Centro para el adelanto tecnológico en salud, Fundación Oswaldo Cruz, Río de Janeiro, Brasil. $₫$ fabio.zicker@gmail.com
}

la pertinencia y el potencial de investigación para abordar los retos de salud de la región. Es ampliamente reconocida la desconexión existente entre la investigación académica y las necesidades de atención de salud en la región. ${ }^{6}$ El financiamiento general destinado a la ciencia y la infraestructura de investigación ha sido vulnerable a la inestabilidad social, económica y política. El financiamiento de la ciencia ha sido una de las primeras partidas presupuestarias gubernamentales que se eliminan ante la presión fiscal. ${ }^{7}$ En este contexto, se han realizado algunas iniciativas para aumentar la capacidad y la pertinencia de la investigación en salud en la región, y en el presente artículo se analizan los éxitos, los obstáculos y las enseñanzas extraídas de algunas de estas iniciativas.

\section{FORTALECIMIENTO DE LOS SISTEMAS NACIONALES DE INVESTIGACIÓN EN SALUD}

El reconocimiento mundial cada vez mayor de la importancia de los programas nacionales de investigación en

\section{MENSAJES CLAVE}

- Pese a la inestabilidad social, económica y política, América Latina y el Caribe ha aumentado su inversión en investigación y desarrollo, y ha triplicado las publicaciones relacionadas con la salud en los últimos años.

- Fomentar los productos de la investigación con pertinencia para la salud pública requiere mecanismos de priorización, fortalecimiento de los sistemas nacionales de investigación y ampliación de la colaboración entre la comunidad académica y las instancias de ejecución de la salud pública.

- La investigación sobre la implementación desempeña un papel importante en el mejoramiento del desempeño de los sistemas de salud.

- La inversión sostenible en infraestructura y recursos humanos es necesaria para fortalecer la contribución de la investigación a la salud pública.

\footnotetext{
Asesor principal para la Promoción y Desarrollo de la Investigación. Organización Panamericana de la Salud/Organización Mundial de la Salud, Washington, D.C. Estados Unidos.
}

\footnotetext{
Asesor principal para la Investigación Mundial en Salud. Centro para Salud Mundial, Instituto Nacional del Cáncer, Institutos Nacionales de Salud, Bethesda, MA, Estados Unidos.
} 
salud en el mejoramiento de los sistemas de salud impulsó al Consejo de Investigación en Salud para el Desarrollo y la Organización Panamericana de la Salud (OPS) a realizar una encuesta sobre los sistemas de investigación en 14 países de la región en el 2008. En ese momento, solo seis países contaban con gobernanza y estructuras de gestión de la investigación establecidas formalmente, dirigidas por el ministerio de salud o el ministerio de ciencia y tecnología, y solo nueve países referían haber fijado las prioridades de la investigación nacional en salud. ${ }^{8}$ Los resultados se analizaron en la primera Conferencia Latinoamericana sobre Investigación e Innovación para la Salud del 2009 e influyeron en la Política de investigación para la salud de la OPS. ${ }^{3}$

Uno de los principales objetivos de la política de la OPS fue establecer prioridades nacionales de investigación en salud. Un examen realizado en el 2014, cinco años después de establecerse la política, indicó un aumento del número de países de América Latina y el Caribe que contaban con agendas de investigación. Catorce de los 37 países incluidos en el examen tenían agendas de investigación, con amplias variaciones en cuanto a los objetivos, los contenidos, los métodos y las esferas estratégicas. ${ }^{9}$ El estudio destacó la necesidad de que los ministerios de salud y los interesados directos en toda la región llevasen a cabo ejercicios de definición de las prioridades nacionales de investigación mediante métodos sistemáticos.

\section{CONVERGENCIA DE LAS PRIORIDADES DE INVESTIGACIÓN}

Los ministerios de salud y de ciencia y tecnología de América Latina y el Caribe han aumentado en forma sostenida su compromiso con los sistemas de atención de salud, mediante la definición de prioridades y la promoción de investigaciones de salud pertinentes. Se han establecido iniciativas de investigación como EVIPNet (Red de Políticas Basadas en la Evidencia) ${ }^{10}$ y SORT IT (programa de capacitación e investigación operativa $)^{11}$ en los ministerios de salud de diez países. La OPS y la Organización Mundial de la Salud (OMS) han facilitado la capacitación, la creación de portales web y la introducción de métodos para evaluar la evidencia. El objetivo de estos programas es empoderar a los encargados de formular políticas, los investigadores y los encargados de la ejecución para subsanar la brecha entre la generación de los conocimientos y su utilización en las políticas. Los puntos de interés centrales han sido el acceso a la atención de salud maternoinfantil de gran calidad, la prevención $y$ el control de las enfermedades infecciosas, el diagnóstico y el tratamiento de la tuberculosis, y el acceso a los medicamentos costosos. Sin embargo, en la práctica persiste la pugna de intereses entre la aplicación de los conocimientos actuales y la generación y la puesta a prueba de nuevos conocimientos y evidencia. ${ }^{3}$

Con el propósito de promover la investigación sobre algunas brechas específicas de los conocimientos, el programa brasileño de investigación para el sistema de salud unificado ha descentralizado el financiamiento de la investigación (US\$ 100 millones en más de 15 años), el desarrollo de tecnologías y la innovación mediante convocatorias para presentar propuestas de investigación en temas prioritarios. $^{12} \mathrm{El}$ proceso ha incluido ejercicios locales de determinación de prioridades gestionados por fundaciones estatales para apoyar la ciencia y la tecnología, en los cuales han participado los encargados de formular políticas, los encargados de la ejecución, los investigadores y en algunas ocasiones las asociaciones de pacientes, con el objeto de proponer agendas de investigación de interés regional. Esta estrategia ha resultado útil para aumentar la demanda de investigación sobre los problemas prioritarios, como la evaluación de tecnologías sanitarias y la gestión de la atención de salud en el lugar de atención, por ejemplo, las pruebas, el diagnóstico y el tratamiento de la tuberculosis en un mismo centro de salud.

\section{MEJORA DE LA CALIDAD Y LOS ESTÁNDARES DE LA INVESTIGACIÓN}

Algunas de las iniciativas más avanzadas de la región encaminadas a fortalecer la calidad de la investigación y sus estándares se encuentran en el Caribe. Las instituciones académicas del Caribe, en colaboración con la Red Internacional de Epidemiología Clínica y la Colaboración Cochrane, han promovido la capacitación para mejorar la calidad de las investigaciones en salud pública. El punto central ha sido producir revisiones sistemáticas, métodos de planificación y evaluación de los proyectos, buenas prácticas clínicas y normas de ética de la investigación. Las iniciativas aprovecharon los programas de becas patrocinados por el programa de nuevos líderes para las Américas, con el apoyo adicional de las universidades de Ottawa, de las Indias Occidentales y Johns Hopkins. Se han capacitado hasta el presente más de 300 becarios. Otras iniciativas en la región, como la división del Caribe del Centro Cochrane de Estados Unidos que se estableció en el 2013 en Jamaica y el Organismo de Salud Pública del Caribe (CARPHA) también imparten capacitación sobre síntesis y traducción de los conocimientos.

Un informe de la evaluación y el impacto de las actividades de fortalecimiento de la capacidad en el Caribe durante el último decenio documentó el progreso realizado en la formación de equipos y la elaboración de marcos institucionales de investigación. ${ }^{13}$ En el informe se reconoció la necesidad de promover la participación colaborativa entre los investigadores y los profesionales de salud, y de optimizar los recursos humanos y económicos mediante asociaciones entre las instituciones del Caribe y de América del Norte y las iniciativas mundiales.

\section{FOMENTO DEL LIDERAZGOY LA FORMACIÓN DE REDES}

Las redes de investigación son una manera eficiente de asociar diferentes disciplinas y pericias con el fin de abordar problemas pertinentes de salud pública y promover la investigación de gran calidad. En un informe de la OMS ${ }^{14}$ se indicó que la eficacia de una colaboración se relaciona con las metas compartidas entre los miembros de la red, una estructura clara de gobernanza, un liderazgo dinámico, buenas prácticas de gestión, recursos sostenibles y una comunicación eficaz.

Se ha utilizado el análisis de redes sociales con el objeto de mapear las relaciones entre los miembros de las redes de investigación y de reconocer a los miembros centrales e influyentes. ${ }^{15}$ Un examen de la red de investigación sobre el dengue puso en evidencia la distribución geográfica desigual de las actividades de investigación y la casi ausencia de participación del sector privado, lo cual constituye una brecha estratégica importante en los esfuerzos encaminados a desarrollar nuevos enfoques para afrontar la epidemia cada vez mayor del dengue. ${ }^{16}$ 
Recuadro 1. Respuesta de la investigación ante la epidemia de síndrome congénito por el virus del Zika en Brasil

En la respuesta ante la epidemia de la infección por el virus del Zika del 2016 participaron los sistemas nacionales y estatales de vigilancia, la comunidad científica y la OPS. Se movilizó el financiamiento y el apoyo político, y las expectativas de los prestadores de atención de salud y del público en general eran altas.

Se creó un grupo de investigación interinstitucional sobre la epidemia de microcefalia, que llevó a cabo un estudio de casos y testigos con el fin de explorar los posibles factores de riesgo asociados con este aumento de la incidencia. ${ }^{17}$ Estos resultados 1 levaron a que se realicen las pruebas de la infección por el virus del Zika en los recién nacidos de 2 días de edad.

Las redes sociales ayudaron a alertar acerca del problema, pero también difundieron rumores sin fundamento que causaron confusión acerca del origen del virus, la transmisión por vectores y la posible propagación de la enfermedad. La declaración del estado de emergencia por parte de las autoridades brasileñas y la OMS fue un punto decisivo para movilizar al sector de la salud pública, la sociedad civil y la comunidad académica. La comunidad científica desempeñó una función central en la descripción del síndrome congénito del Zika, la formulación de su etiología y la obtención de la evidencia para fundamentar las decisiones políticas. El resultado de estas iniciativas fue la creación de la Red Latinoamericana de Preparación para el Zika (https:/ / zikaplan.tghn.org).

Recuadro 2. Mejora del tamizaje del VPH en América Latina, el estudio ESTAMPA

El estudio ESTAMPA está dirigido por el Centro Internacional de Investigaciones sobre el Cáncer de la OMS en colaboración con diversos ministerios de salud de América Latina y con el apoyo del Instituto Nacional del Cáncer de Estados Unidos y la OPS. Se prevé incluir hasta 50000 participantes con el objeto de evaluar la costo-efectividad de la ejecución de diferentes métodos de selección para detectar la infección por el virus del papiloma humano (VPH).

La realización de este amplio estudio multinacional ha resultado difícil y compleja, dado que incluye una red de científicos, instituciones y gobiernos. La experiencia indica que los mejores resultados y la mejor calidad de los datos dependen en gran parte de la planificación eficaz, el conocimiento de la carga de enfermedad local, el análisis de costos de despliegue en los centros del estudio, el análisis de las posibilidades locales para ampliar la escala de aplicación, la participación de las comunidades y la gestión interdisciplinaria.

Dado que la investigación sobre la ejecución es una disciplina nueva y que las oportunidades de capacitación en el marco de una institución académica aún deben ampliarse, la OMS y el Programa Especial de Investigaciones y Enseñanzas sobre Enfermedades Tropicales crearon un curso abierto en línea, dirigido a los investigadores y los encargados de ejecutar los programas de salud. El objetivo del curso es situar la investigación sobre la ejecución como un método importante para llevar a la práctica las innovaciones en las políticas al impartir capacitación de fácil acceso en América Latina y el Caribe y otras regiones de la OMS. ${ }^{19}$

Se puede aprender mucho de la reciente epidemia de microcefalia causada por el virus del Zika en el nordeste de Brasil. El liderazgo y la formación de redes fueron esenciales en el intercambio de la información, la generación y la difusión de datos nuevos, y en la definición de las líneas estratégicas de investigación como respuesta a la emergencia. La rapidez del descubrimiento de su etiología y de la formulación del manejo corriente de los casos se logró gracias a los enfoques interdisciplinarios y la colaboración interinstitucional del grupo de investigación sobre la epidemia de microcefalia (recuadro 1).

\section{INVESTIGACIÓN SOBRE LA GESTIÓN DE LOS PROGRAMAS}

Uno de los mensajes más importantes de la segunda Conferencia Latinoamericana sobre Investigación e Innovación para la Salud que tuvo lugar en el $2011^{6}$ fue que la innovación en la investigación no se mide según la complejidad de una intervención o de la solución de un problema. La investigación innovadora puede conducir a soluciones sencillas que abordan las grandes cargas sociales y económicas de los sistemas de salud. Esta conclusión tiene una pertinencia especial en la investigación sobre la implementación.

La investigación sobre la implementación es una esfera que cobra un interés cada vez mayor en América Latina y el Caribe, orientada hacia la comprensión de los determinantes del éxito y también de los obstáculos a la introducción o la ampliación sostenibles de las estrategias y las tecnologías de salud. ${ }^{18}$ En la actualidad, la investigación sobre la implementación no se utiliza plenamente en la región, aunque hay algunos buenos ejemplos como el estudio multicéntrico
ESTAMPA. Este estudio se diseñó con el objeto de evaluar el desempeño y la costo-eficacia de diferentes algoritmos y procedimientos de tamizaje del VPH (recuadro 2).

\section{CONCLUSIÓN}

La investigación cumple una función importante en el desarrollo sostenible al mejorar el desempeño de los sistemas de salud y su repercusión sobre la salud pública. La ciencia, la tecnología y la innovación forman parte de varios objetivos de desarrollo sostenible de las Naciones Unidas. ${ }^{20}$ América Latina y el Caribe está comprometida a alcanzar estas metas, pero el aumento necesario de la investigación en salud en la región es todavía heterogéneo. ${ }^{21}$ Los sistemas nacionales de salud e investigación deben examinar y armonizar las metas, fortalecer la infraestructura y supervisar 
los mecanismos para recoger datos fidedignos que evalúen el progreso en el desarrollo social.

Contribución de los autores. Todos los autores contribuyeron a la planificación y la redacción del manuscrito original. Celina Turchi y Enrique Vázquez contribuyeron con el ejemplo del Zika en el recuadro 1 y Rolando Herrero y Maribel Almonte aportaron el ejemplo del estudio del recuadro 2 .

Conflicto de intereses. Después de haber leído y comprendido la política de The BMJ sobre el conflicto de intereses, los autores declaran no tener ningún conflicto de intereses. FZ recibió apoyo de investigación del Consejo Nacional Brasileño para el Desarrollo Científico y Tecnológico (CNPq), subvenciones \#458100/2014-5 y \#440231/2015-9.

Procedencia del artículo y revisión por pares. Comisionado; revisión por pares externa.

Declaración. Las opiniones expresadas en este manuscrito son responsabilidad de los autores y no reflejan necesariamente los criterios ni la política de la RPSP /PAJPH y/o de la OPS/OMS.

\section{REFERENCIAS}

1. Organización Mundial de la Salud. Strategy on research for health. 2012. http:// www.who.int/phi/WHO_Strategy_on research_for_health.pdf

2. Ghaffar A, Jsselmuiden I, Zicker F. Changing mindsets: research capacity strengthening in low- and middle-income countries. OMS, 2010. http://www.who int/tdr/publications/documents/changing mindsets.pdf

3. Organización Panamericana de la Salud/ Organización Mundial de la Salud. Política de investigación para la salud. $49{ }^{\circ}$ Consejo Directivo, 61. ${ }^{a}$ sesión del Comité Regional de la OMS para las Américas. 2009. https: / / www.paho.org/hq/images/stories/KBR /Research/politica\%20de\%20investiga cion $\% 20$ para $\% 201$ la $\% 20$ salud.pdf

4. Banco Mundial. Research and development expenditure. https://data.worldbank .org/indicator/GB.XPD.RSDV.GD.ZS ?locations $=\mathrm{XJ}$

5. Scimago Journal \& Country Rank. Disponible en http://www.scimagojr.com (consultado el 16 de octubre del 2017).

6. Consejo de Investigación en Salud para el Desarrollo (COHRED). Informe de la segunda Conferencia Latinoamericana sobre Investigación e Innovación para la Salud, Ciudad de Panamá, 2011. http:// www.cohred.org/wp-content/uploads /2011/10/COHRED_LAT_SP_web.pdf.

7. Ciocca DR, Delgado G. The reality of scientific research in Latin America; an insider's perspective. Cell Stress Chaperones 2017;22:847-52. doi:10.1007/ s12192-017-0815-8

8. Alger J, Becerra-Posada F, Kennedy A, Martinelli E, Cuervo LG. Sistemas nacionales de investigación para la salud en América Latina: una revisión de 14 países [National health research systems in Latin America: a 14-country review]. Rev Panam Salud Publica 2009;26:447-57.
9. Becerra-Posada F, de Snyder NS, Cuervo LG, Montorzi G. Agendas de investigación priorizadas: un recurso estratégico para la salud en América Latina [Priority research agendas: a strategic resource for health in Latin America]. Rev Panam Salud Publica 2014;36:361-7.

10. Organización Mundial de la Salud. EVIPnet. http:/ / www.who.int/evidence/en/

11. Organización Mundial de la Salud, Programa Especial de Investigaciones y Enseñanzas sobre Enfermedades Tropicales. SORT IT. https://www.who.int/tdr /capacity/strengthening/sort/en/.

12. Ministério da Saúde Brasil. Programa de Pesquisa para o SUS (PPSUS). 2017. http://www.brasil.gov.br/saude/2017/02 /ministerio-da-saude-garante-80-dos -recursos-para-pesquisa-no-setor-no-pais

13. Organización Panamericana de la Salud /Organización Mundial de la Salud. Report on strengthening research capacities for health in the Caribbean, 2007-2017. 2017. http://iris.paho.org/xmlui/handle $/ 123456789 / 34342$

14. Organización Mundial de la Salud, Programa Especial de Investigaciones y Enseñanzas sobre Enfermedades Tropicales. Key enabling factors in effective and sustainable research networks. 2016. http:/ / www.who.int/tdr/publications /research-networks/en/

15. Fonseca BdeP, Sampaio RB, Fonseca MVA, Zicker F. Co-authorship network analysis in health research: method and potential use. Health Res Policy Syst 2016;14:80. doi:10.1186/s12961-016-0104-5

16. Fonseca BdeP, Zicker F. Dengue research networks: building evidence for policy and planning in Brazil. Health Res Policy Syst 2016;14:34. doi:10.1186/s12961-016-0104-5

17. de Araújo TVB, Rodrigues LC, de Alencar Ximenes RA, et al, en nombre del grupo de investigaci'on de la epidemia de
Serie Fortalecimiento de la investigación para la salud en las Américas. Este artículo es parte de una serie propuesta por la Organización Panamericana de la Salud (OPS) y comisionada por The BMJ, que tuvo a cargo la revisión por pares y la edición, y tomó la decisión sobre la publicación de los artículos sin participación de la OPS. Los gastos de publicación en acceso abierto de la serie original en inglés fueron financiados por la OPS. Acceso a la serie original en inglés en www. bmj.com/health-research-americas: acceso a la serie en español y portugués en https://www.paho.org/journal/es /numeros-especiales / fortalecimiento -investigacion-para-salud-americas. microcefalia. Ministerio de salud de Brasil, Organización Panamericana de la Salud, Instituto de Medicina Integral Profesor Fernando Figueira, departamento de salud de Pernambuco. Association between Zika virus infection and microcephaly in Brazil, January to May, 2016: preliminary report of a case-control study. Lancet Infect Dis 2016;16:1356-63. doi:10.1016/ S1473-3099(16)30318-8

18. Peters DH, Adam T, Alonge $\mathrm{O}$, Agyepong IA, Tran N. Implementation research: what it is and how to do it. BMJ 2013;347:f6753. doi:10.1136/bmj.f6753.

19. Organización Mundial de la Salud Programa Especial de Investigaciones y Enseñanzas sobre Enfermedades Tropicales. New online course on implementation research under development. TDR News 2015. http://www.who.int /tdr/news/2015/new-online-course/en/

20. Naciones Unidas. Objetivos de desarrollo sostenible: 17 objetivos para transformar nuestro mundo. https://www.un.org /sustainabledevelopment/es/

21. Becerra-Posada F, Minayo M, Quental C, de Haan S. National research for health systems in Latin America and the Caribbean: moving towards the right direction? Health Res Policy Syst 2014;12:13. doi:10.1186/1478-4505-12-13

Forma de citar (artículo original): BMJ 2018;362:k2492 doi: http:/ /dx.doi.org/10.1136/bmj.k2492

Manuscrito original en inglés publicado el 16 de julio de 2018. 